\title{
Statistik schön einfach - Eine Einführung in 50 Bildern
}

Thomas Spiegler. Verlagsgruppe Beltz Juventa, Weinheim Basel, 2020. 138 Seiten, ISBN 978-3-7799-6306-6, $€ 17,95$

Warum rezensiere ich ein Statistikbuch für „Lernen und Lernstörungen"? Die Antwort steckt im Titel dieses Buches. „Statistik schön einfach“ verspricht, was viele andere Statistikbücher nicht leisten: Eine anschauliche Einführung in statistische Themen anhand alltagsnaher Bilder. Aus meiner eigenen Lehrtätigkeit in statistischen Methoden kenne ich zu gut die Frustration meiner Kursteilnehmer_innen, wenn statistisches Wissen zu abstrakt vermittelt wird. Aber auch für Lerntherapeut_innen und Lehrpersonen kann es oft eine Herausforderung darstellen, die statistischen Ergebnisse wissenschaftlicher Studien $\mathrm{zu}$ verstehen und $\mathrm{zu}$ interpretieren. Thomas Spiegler, Professor für Soziologie und empirische Sozialforschung an der Theologischen Hochschule Friedensau, versucht mit diesem Buch Abhilfe zu schaffen.

„Statistik schön einfach“ präsentiert die Grundlagen der Statistik, angefangen von einer Erläuterung, was Statistik überhaupt macht, über Erklärungen der wichtigsten Maße mit denen Daten beschrieben werden, bis hin zur Erkennung und Beschreibung von Zusammenhängen. Anschließend folgt eine kurze Klärung hilfreicher Begriffe sowie ein Anhang mit vertiefenden Details und weiteren Beispielen. Wer in diesem Buch mathematische Gleichungen sucht, wird keine finden. Stattdessen erwarten die Leserin oder den Leser wie auf dem Titelblatt versprochen 50 anschauliche Darstellungen, deren Inhalte anhand kurzer Texte erläutert werden. So wird zum Beispiel die Auszählung von Häufigkeiten anhand der Anzahl von Mädchen und Jungen in einem Foto einer Schulklasse erklärt.
Wie vom Autor selbst im Vor wort beschrieben, kann un will „Statistik schön einfach“ keine üblichen Lehrbücher ersetzen, sondern „(...) kreativ ergänzen und gewaltfrei dazu hinführen". Dieser Eindruck bestätigte sich mir beim Lesen. Die Texte und Bilder sind gut aufeinander abgestimmt und sowohl verständlich verfasst als auch ansprechend gestaltet. Die wichtigsten Begriffe aus jedem Text werden am Ende der Seite erneut aufgeführt und können im Register nachgeschlagen werden. Übungsmaterial zur Festigung der Inhalte enthält das Buch allerdings nicht. Zudem werden zugunsten der Verständlichkeit keine komplexeren statistischen Analysemethoden beschrieben, und müssen daher anderswo recherchiert werden.

Was dem Autor aber gelingt, ist statistische Konzepte anhand anschaulicher Beispiele und Bilder auch für Laien greifbar zu machen. Positiv fällt zudem die Verwendung möglichst einfacher, alltagsnaher Sprache auf, was die Lektüre entsprechend kurzweilig macht. All jenen, die sich mit wissenschaftlichen Texten auseinandersetzen, kann dieses Buch dabei helfen, zumindest die grundlegenden darin enthaltenen statistischen Begriffe zu verstehen. Abschließend kann ich mich der Aussage des Autors aus dem Vorwort anschließen: „Es ist ein Buch für Menschen, die Statistik brauchen, aber sie (noch) nicht mögen, und auch für jene, die solchen Menschen Statistik verständlich machen möchten." In mein nächstes Statistik-Seminar werde ich es auf jeden Fall mitbringen. 